\title{
Vulnerabilidad de los bosques naturales en el Chocó biogeográfico colombiano: actividad minera y conservación de la biodiversidad
}

\author{
Vulnerability of native forests in the Colombian Chocó: mining and biodiversity conservation
}

\author{
Hamleth Valois-Cuesta ${ }^{a^{*}}$, Carolina Martínez-Ruiz ${ }^{\mathrm{b}}$ \\ *Autor de correspondencia: a Universidad Tecnológica del Chocó, Facultad de Ciencias Naturales, \\ Programa de Biología, Quibdó, Colombia; tel.: 57-4-6171616, hamlethvalois@gmail.com \\ ${ }^{\mathrm{b}}$ Universidad de Valladolid, E.T. S. de Ingenierías Agrarias, Instituto Universitario de Investigación \\ en Gestión Forestal Sostenible UVa-INIA, Avda. de Madrid 44, 34071 Palencia, España.
}

\begin{abstract}
SUMMARY
Chocó is a region with high biodiversity and impacted by gold and platinum mining. This study determined the potential vulnerability of forests of Chocó to the loss of plant species in a context of mining activity. Field trips to different mining municipalities of the Chocó region were conducted in order to document the techniques of mineral extraction and the miners' perception on the performance and the environmental impact of mining in their territories. From data on gold and platinum production (1990-2012), the number of mining titles (2012-2013), number of mines and type of machinery used (2008-2012), the indices of mining activity (IAMAR) and relative mining production (IPMAR) were determined for all the territorial entities of Chocó. Additionally, the number of endemic species and threatened species was estimated and used to calculate the potential floristic vulnerability index (IVFP) regarding loss of species. The IVFP varied significantly between municipalities and subregions of the Chocó territory. The Municipalities with more IVFP were Condoto (9.43\%), Istmina (7.75 \%), Nóvita (7.74 \%), Quibdó (7.64 \%) and San José del Palmar (6.56 \%). At the subregional level, San Juan (54.8\%) had the highest IVFP. Mining in Chocó threats strategic ecosystems of global importance for the conservation of biodiversity and highlights the need for measures to prevent and mitigate the loss of species in areas with high vulnerability due to mining.
\end{abstract}

Key words: ecological vulnerability index, mining production index, mining activity index, biological conservation, gold and platinum mining.

\section{RESUMEN}

El Chocó es una región con alta biodiversidad e impactada por la minería de oro y platino. En este trabajo se determinó la vulnerabilidad potencial de los bosques del Chocó para la pérdida de especies vegetales debido a la actividad minera. En distintos municipios mineros del territorio chocoano se documentaron las técnicas de extracción de minerales y la percepción de los mineros sobre la minería en sus territorios. A partir de datos de producción de oro y platino (1990-2012), títulos mineros (2012-2013) y número de minas y maquinaria usada (2008-2012) se propusieron los índices de actividad minera (IAMAR) y de producción minera relativa (IPMAR) en todos los entes territoriales del Chocó; ambos índices se aplican en este estudio por primera vez. Además se cuantificó el número de especies de distribución restringida y el número de especies amenazadas como factores de vulnerabilidad. Con esas cuatro variables se determinó el índice de vulnerabilidad florística potencial para la pérdida de especies (IVFP), que varió significativamente entre municipios y subregiones del territorio chocoano. Los municipios con mayor IVFP fueron Condoto $(9,43 \%)$, Istmina (7,75 \%), Nóvita (7,74 \%), Quibdó (7,64 \%) y San José del Palmar (6,56 \%). A nivel subregional, el San Juan (54,8 \%) presentó el mayor IVFP. La minería en el Chocó amenaza ecosistemas estratégicos de importancia mundial para la conservación de la biodiversidad, lo que hace necesario establecer medidas para prevenir y mitigar la perdida de especies en territorios con alta vulnerabilidad debido a la actividad minera.

Palabras clave: índice de vulnerabilidad florística, índice de producción minera, índice de actividad minera, conservación biológica, minería auroplatinífera.

\section{INTRODUCCIÓN}

La región del Chocó (Colombia) es reconocida mundialmente por su alta biodiversidad y elevado grado de endemismo: existen registros de 5.976 especies de plantas vasculares (Bernal et al. 2015), 206 de mamíferos, 188 de reptiles, 139 de anfibios, 793 de aves, 196 de peces de agua dulce y 176 de escarabajos (Rangel-Ch 2004a). Además, la región cuenta con importantes yacimientos de oro y platino (Leal 2009), cuya explotación contribuye, en gran medida, a la economía de Colombia.

El Chocó ocupa el primer lugar dentro de la producción colombiana de oro y platino (Agencia Nacional de Minería 2015). A pesar de esta riqueza natural, el Chocó es una 
región con poco desarrollo económico, donde convergen la necesidad de conservar la naturaleza, como fuente de bienestar humano, y la necesidad de explotar los minerales del subsuelo, como fuente de empleo y desarrollo económico para la región y el país. En consecuencia, hay que adelantar acciones para hacer de la minería una actividad más amigable con el medio ambiente, pues existe una gran preocupación por la conservación del potencial que posee esta región en cuanto a la oferta de servicios ecosistémicos (Andrade-C 2011).

Esta preocupación se ha intensificado en los últimos años, por el auge que está experimentando la minería de oro y platino en el departamento del Chocó. Empresas mineras y mineros independientes (legales e ilegales) ven en este territorio una oportunidad para consolidar su capital financiero, desconociendo o pasando por alto los graves impactos que la actividad minera produce sobre la biodiversidad de los bosques tropicales (Ramírez-Moreno y Ledezma-Rentería 2007, Andrade-C 2011). Sin embargo, el conocimiento de la vulnerabilidad de los bosques tropicales frente a la minería es vital, para conseguir el ordenamiento de territorios en contextos geográficos ricos en biodiversidad, pero marcados tradicionalmente por una economía basada en la actividad minera.

Considerando que la minería es un factor de riesgo para la conservación de la vida silvestre en regiones de alta biodiversidad, surge la pregunta ¿Cómo cambia el grado vulnerabilidad de los bosques naturales en distintos territorios frente a la minería? La hipótesis de partida es que el grado de vulnerabilidad para la pérdida de especies en los bosques de un determinado territorio depende del grado de incidencia minera (actividad y producción) y de la sensibilidad de la biodiversidad (especies raras y/o amenazadas) que alberga.

En este marco se plantea este trabajo, cuyo objetivo es determinar la vulnerabilidad potencial de los bosques naturales del Chocó, a nivel municipal y subregional, frente a la actividad minera auroplatinífera como factor de riesgo para la pérdida de especies.

\section{MÉTODOS}

Área de estudio. El departamento del Chocó (0400'50"$08^{\circ} 41^{\prime} 32^{\prime \prime}$ N y $76^{\circ} 02^{\prime} 57^{\prime \prime}-77^{\circ} 53^{\prime} 38^{\prime \prime}$ O) tiene una extensión de $44.530 \mathrm{~km}^{2}$ y está compuesto por 30 municipios agrupados en cinco subregiones (Poveda-M et al. 2004): 1) Alto Atrato, pie de monte de la Cordillera Occidental de Colombia al oeste del departamento del Chocó; 2) Medio Atrato, llanuras aluviales del río Atrato en la zona central del departamento; 3) Bajo Atrato, llanuras aluviales y zonas estearinas del Océano Atlántico al norte del departamento; 4) Litoral del Pacífico, llanuras aluviales con zonas estearinas del Océano Pacífico al suroeste del departamento; y 5) San Juan, llanuras aluviales y colinas bajas en la cuenca del río San Juan al sureste del departamento.

El Chocó pertenece a la región del Chocó biogeográfico colombiano que se caracteriza por su alta pluviosidad, con valores anuales de hasta $13.670 \mathrm{~mm}$, una temperatura promedio anual de $26^{\circ} \mathrm{C}$ y una humedad relativa del $80 \%$ (Poveda-M et al. 2004). La región cuenta con selvas de alta riqueza, diversidad y variedad de endemismos (Rangel-Ch y Rivera-Díaz 2004, Bernal et al. 2015).

La biodiversidad del territorio chocoano ha sido fuente de bienes y servicios para la subsistencia de los pueblos asentados en la región. A pesar de ello, en la actualidad la minería ocupa un lugar importante en la economía local (Leal 2009).

Fuentes de datos. Se realizaron salidas de campo entre los años 2013 y 2014 a los municipios de Condoto, Istmina, Tadó, Unión Panamericana y Río Quito (Chocó), con el fin de documentar las técnicas de explotación minera empleadas y los impactos que estas generan sobre la selva. Además, se entrevistaron 65 mineros, con la finalidad de obtener información sobre su percepción en relación a los aspectos económicos y ambientales de la minería. En concreto, se les preguntó sobre qué técnica de extracción minera (retro, draga o barequeo) genera más beneficio económico y cuál provoca más daño ambiental.

Posteriormente, con el fin de identificar la incidencia de la actividad minera en todos los entes territoriales del Chocó, se extrajeron datos oficiales de títulos mineros otorgados (2012-2013) y producción de oro y platino (1990-2012), disponibles en el Sistema de Información Minero de Colombia (Agencia Nacional de Minería 2015). Además, se solicitaron datos a la Corporación Autónoma Regional para el Desarrollo Sostenible del Chocó sobre el número de minas y maquinaria (retroexcavadoras $\mathrm{y} / \mathrm{o}$ dragas de succión) activa en el territorio chocoano (20082012) (CODECHOCO 2012). A partir de estos datos se propusieron dos índices: (1) el índice de producción minera auroplatinífera relativa (IPMAR) y (2) el índice de actividad minera auroplatinífera relativa (IAMAR):

$$
\mathrm{IPMAR}=\mathrm{PRO}+\mathrm{PRP}
$$

Donde:

$\mathrm{PRO}=$ producción relativa de oro.

$\mathrm{PRP}=$ producción relativa de platino.

$$
\mathrm{IAMAR}=\mathrm{NRMI}+\mathrm{NRMA}+\mathrm{NRTMO}
$$

\section{Donde:}

$\mathrm{NRMI}=$ número relativo de minas.

NRMA = número relativo de máquinas activas.

$\mathrm{NRTMO}=$ número relativo de títulos mineros otorgados.

Para ambos índices, los valores relativos para un determinado municipio o subregión se calcularon dividiendo el valor absoluto de la variable para ese municipio o subregión entre el sumatorio de los valores obtenidos para todos los municipios o subregiones y multiplicando por 100. En ambos casos, a mayor valor del índice mayor fue la incidencia de la minería en el ente territorial. 
Adicionalmente, con el fin de verificar el nivel de conocimiento de los bosques de cada municipio y subregión en términos de riqueza y composición florísticas, se cuantificaron los registros de especies vegetales citados en el catálogo de Rangel-Ch et al. (2004), y se revisaron 80.513 registros de colecciones biológicas del Chocó en el Sistema de Información sobre Biodiversidad de Colombia ( $\mathrm{SiB}$ Colombia 2015). Para determinar el número de especies vegetales por municipio y subregión se consideraron únicamente los registros (distribución geográfica) que estuvieran claramente circunscritos a un determinado ente territorial $(\mathrm{N}=45.225)$. Aquellos registros con ambigüedad en su localización no fueron cuantificados. El número total de especies vegetales para el departamento se determinó sobre la base del Catálogo de Plantas y Líquenes de Colombia (Bernal et al. 2015).

Dado que la cantidad, tanto de especies de distribución restringida como en peligro de extinción, puede ser considerado como un factor de vulnerabilidad frente a las perturbaciones antrópicas, se obtuvieron: la lista de especies amenazadas, a partir de los inventarios de Rangel-Ch. (2004b), y la lista de especies nativas de Colombia restringidas en su distribución al departamento de Chocó, a partir del Catálogo de Plantas y Líquenes de Colombia (Bernal et al. 2015). Con estas dos listas elaboradas, el número de especies pertenecientes a cada municipio y subregión del Chocó se cuantificó usando los datos de distribución geográfica de las especies de interés citadas en el Catálogo de espermatofitos del Chocó biogeográfico (Rangel-Ch. et al. 2004) y las bases de datos: SIB-Colombia (SIB-Colombia 2015), Tropicos (Tropicos 2015) y Herbario virtual de New York Botanical Garden (NYBG 2015). En el caso de las especies de distribución restringida, solo se consideraron 123 especies nativas de Colombia, restringidas al Chocó, pertenecientes a las familias: Rubiaceae, Orchidaceae, Melastomataceae, Piperaceae, Araceae, Asteraceae, Bromeliaceae, Fabaceae, Clusiaceae, Poaceae, Gesneriaceae, Euphorbiaceae, Arecaceae, Moraceae y Bombacaceae, por ser las familias más diversificadas, de más amplia distribución y con mayor número de especies restringidas al Chocó biogeográfico colombiano (Rangel-Ch y Rivera-Díaz 2004, Bernal et al. 2015).

Finalmente, considerando que altos valores de los índices IAMAR e IPMAR conllevan mayor pérdida de especies vegetales debido a la eliminación de la cubierta vegetal en bosques naturales, y que las especies amenazadas (EA), así como también las especies de distribución restringida (EDR), pueden considerarse como factores útiles para identificar prioridades de conservación (Myers et al. 2000), se determinó para cada uno de los municipios y subregiones el índice de vulnerabilidad florística potencial (IVFP):

$\operatorname{IVFP}=\operatorname{IAMAR}(\%)+\operatorname{IPMAR}(\%)+\mathrm{EA}(\%)+\mathrm{EDR}(\%)$

Teniendo en cuenta que los bosques del Chocó biogeográfico son ricos en especies vegetales por unidad de superficie (aproximadamente 262 especies con diámetros mayores a 2,5 cm en 0,1 ha; Gentry 1986), se asumió que la diferencia en riqueza y diversidad por unidad de superficie entre los entes territoriales estudiados aquí es mínima (Rangel-Ch y Rivera-Díaz 2004). Por tanto, ese factor no se integró como una variable del índice de vulnerabilidad florística potencial, pues se consideró que desde el punto de vista conservacionista todos los territorios tienen el mismo valor. Así, el grado de vulnerabilidad potencial de los bosques de un determinado municipio o subregión aumentó con el porcentaje de actividad minera, de especies en peligro de extinción y/o de especies de distribución restringida. Cuanto mayor fue valor del índice, mayor era el grado de vulnerabilidad potencial de los ecosistemas forestales en cuanto a la pérdida de especies.

Es importante resaltar que el grado de sensibilidad en las estimaciones del índice de vulnerabilidad florística potencial, en cualquier región del mundo, dependerá de la calidad de la información existente sobre incidencia minera y biodiversidad u otras propiedades del ecosistema que se quieran involucrar.

Análisis estadístico. Las diferencias en la percepción socioeconómica y ambiental de los mineros frente a la minería auroplatinífera se evaluaron mediante la prueba Chicuadrado, mientras que las diferencias entre la producción promedio anual de oro y platino en el Chocó, y entre el Chocó y el promedio nacional se analizaron con la prueba $t$ de Student. Para explorar las diferencias en el volumen de producción de oro y platino $\left(\mathrm{kg}\right.$ año $\left.{ }^{-1}\right)$ entre municipios se usó la prueba de Kruskal-Wallis. Un análisis de regresión lineal se empleó para la relación entre IAMAR e IPMAR, y un análisis de componentes principales (ACP) para identificar gradientes subyacentes en la relación entre los entes territoriales y aquellas variables que definen el índice de vulnerabilidad florística potencial.

Todos los análisis se realizaron en el entorno de programación R (R Core Team 2012), excepto el análisis de componentes principales (ACP) que se realizó con el programa CANOCO versión 4,5, empleando las opciones fijadas por defecto (ter Braak y Šmilauer 2002).

\section{RESULTADOS}

Actividad y producción minera. Se identificaron tres tipos básicos de minería auroplatinífera según el tipo de herramientas usadas: 1) minería tradicional con instrumentos artesanales en pequeños caños (figura 1A-B), 2) minería a cielo abierto con retroexcavadoras y motobombas en el seno de los bosques (figura 1C-D) y 3 ) minería con dragas de succión en riveras de ríos y quebradas (figura 1E-F). El $93 \%$ de los mineros encuestados (60 personas) percibe la minería con maquinaria pesada (retroexcavadoras $=43,1 \%$ y dragas de succión 49,2\%) como una actividad más rentable y productiva que la minería artesanal con batea y canalón $\left(7,7 \%\right.$; $\left.\mathrm{Chi}^{2}=19,6, P<0,0001\right)$. Sin 
embargo, estas mismas técnicas mineras son identificadas como las más agresivas ambientalmente (retroexcavadoras $=64,6 \%$, draga $=35,4 \%$ y batea-canalón $=0 \%$; $\left.\mathrm{Chi}^{2}=40,8, P<0,0001\right)$.

Durante el periodo de 23 años comprendido entre 1990 y 2012 , la producción de oro $\left(5.035,3 \pm 1.762,3 \mathrm{~kg}_{\text {año }}{ }^{-1}\right)$ fue mayor que la producción de platino $(1.032,5 \pm 95,7 \mathrm{~kg}$ año $\left.0^{-1}\right)$ en el Chocó $(t=2,3, P=0,03)$. Sin embargo, para ambos metales, la producción neta del Chocó superó la producción media nacional (oro $=1.085,0 \pm 96,5 \mathrm{~kg}^{2}$ año ${ }^{-1}$, $t=2,2, P=0,03$; platino $=75,7 \pm 6,7 \mathrm{~kg}$ año ${ }^{-1}, t=9,9$,
$P<0,0001$; figura 2). Particularmente, la producción de oro aumentó en el Chocó a partir del año 2009, mientras que la producción de platino se mantuvo en el tiempo con fluctuaciones menos marcadas que las del oro, pero superiores a las del promedio nacional (figura 2).

Dentro del territorio chocoano se registró actividad y/o producción de minería auroplatinífera en el 93,5\% de los entes municipales (29 municipios de 30). En 26 de ellos $(83,8 \%)$ se registró producción de oro, mientras que en 21 de ellos $(67,7 \%)$ se registró producción de platino. El volumen de producción de ambos metales $\left(\mathrm{kg}\right.$ año ${ }^{-1}$;
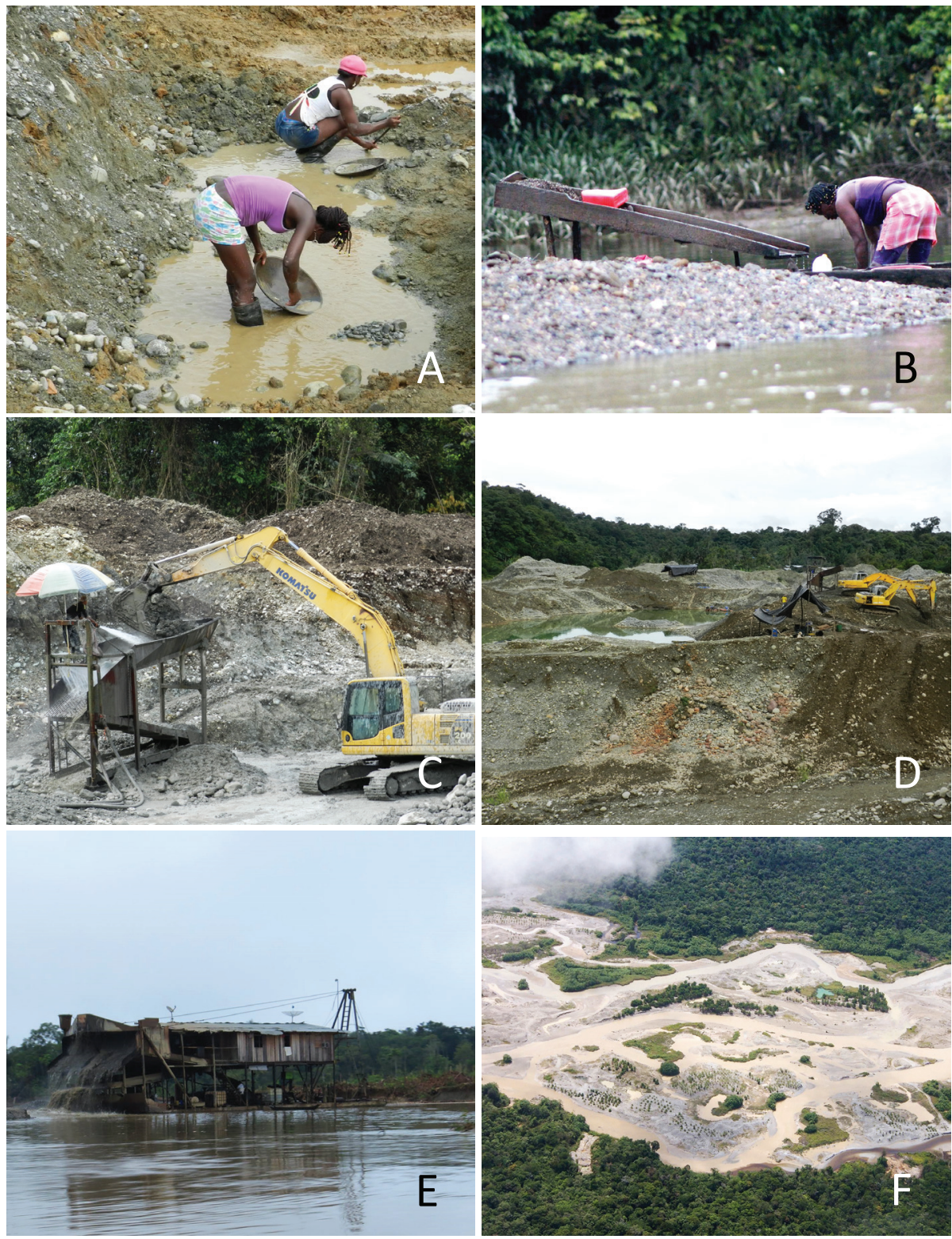

Figura 1. Técnicas para la extracción informal de oro y platino en ecosistemas naturales del Chocó, Colombia: Minería con instrumentos artesanales (A-B), retroexcavadoras y motobombas de alta presión (C-D), y dragas de succión (E-F).

Techniques for the informal extraction of gold and platinum mining in the natural ecosystems of Chocó, Colombia: Mining with handmade instruments (A-B), backhoes and high pressure water pumps (C-D) and suction dredges (E-F). 


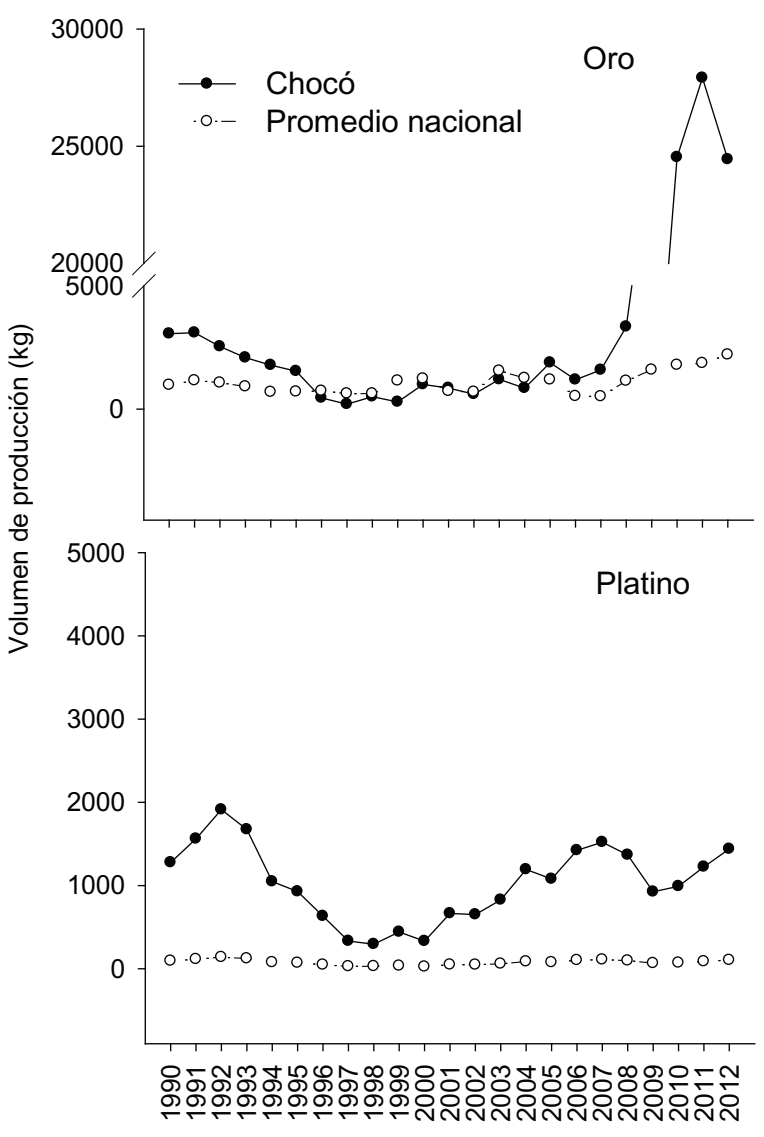

Figura 2. Producción de oro y platino en el departamento del Chocó, en relación a la producción nacional colombiana, periodo 1999-2012.

Gold and platinum production in the department of Chocó, in relation to the Colombian national production, 1999-2012 period.

oro de 2001 a 2012 y platino de 2004 a 2012) difiere significativamente entre municipios (Kruskal-Wallis: oro; $\mathrm{Chi}^{2}=160,3, \mathrm{gl}=26, P<0,0001 ;$ platino; $\mathrm{Chi}^{2}=126,3$, $\mathrm{gl}=20, P<0,0001)$. Condoto $(18,0 \%)$, Nóvita $(13,6 \%)$, Istmina $(10,0 \%)$ y Unión Panamericana $(8,3 \%)$ fueron los municipios con mayor índice de producción minera auroplatinífera (IPMAR) en el Chocó (cuadro 1, figura 3). En conjunto representan el $14 \%$ de los municipios donde se registró minería auroplatinífera y el $50 \%$ de la producción reportada para el Chocó. Los 23 municipios restantes $(85,1 \%)$ aportaron el otro $50 \%$ de la producción reportada para este departamento, y mostraron valores de producción muy variables que van desde $0,0001 \%$ en Bahía Solano hasta 7,1 \% en Medio Baudó (cuadro 1).

Como era de esperar, la producción auroplatinífera en los municipios (IPMAR) no fue independiente de la incidencia de la actividad minera (IAMAR) en aquellos territorios, Así, en términos generales, aquellos municipios que registraron alta producción auroplatinífera también mostraron alta actividad minera (cuadro 1). Al respecto, IAMAR e IPMAR mostraron una relación positiva altamente

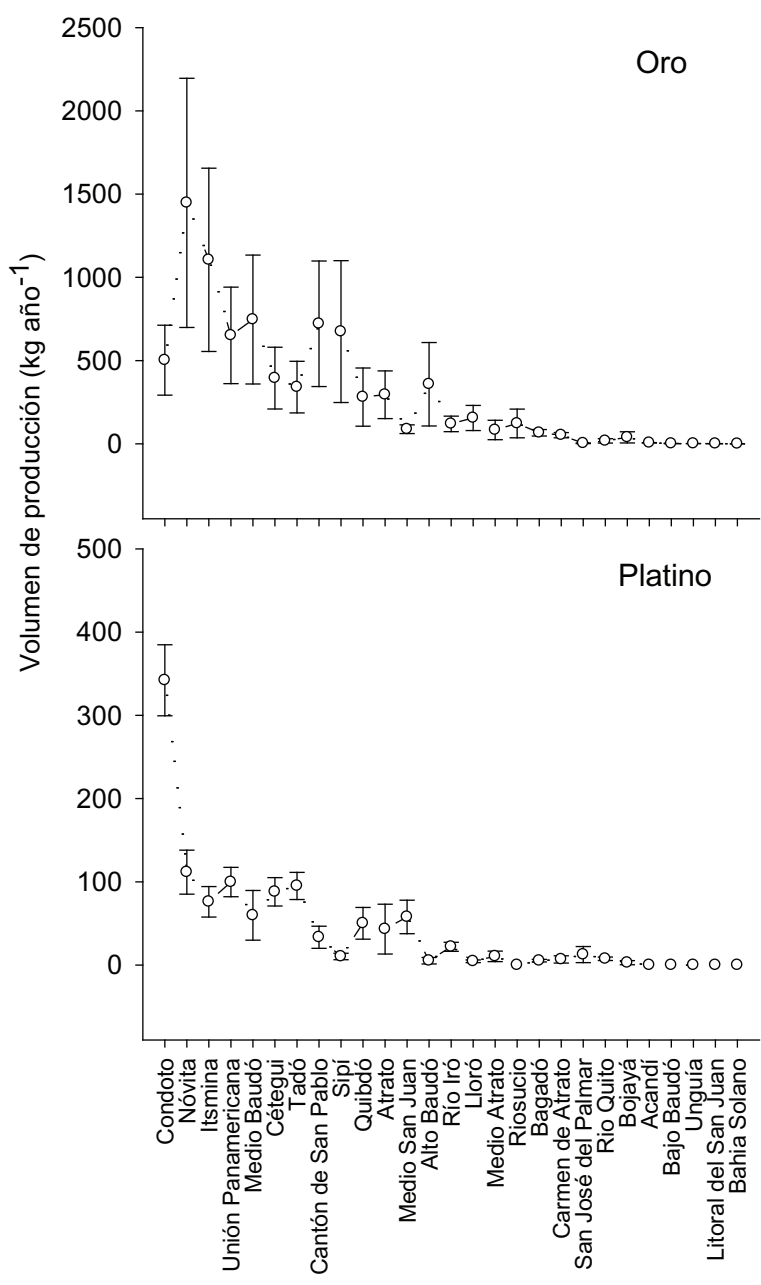

Figura 3. Producción de oro y platino por municipio in Chocó, Colombia. Datos: platino (2004-2012) y oro (2001-2012). Gold and platinum production by municipality in Chocó, Colombia. Data: platinum (2004-2012) and gold (2001-2012).

significativa $\left(\mathrm{r}^{2}=0,53, t_{29}=6,0, P<0,0001\right)$. En general, los municipios con mayor incidencia minera (actividad + producción) fueron Condoto, Nóvita, Istmina, Cantón de San Pablo, Tadó y Unión Panamericana (cuadro 1).

$\mathrm{Al}$ analizar la incidencia de actividad minera (IAMAR) y producción (IPMAR) auroplatinífera a escala subregional, se encontraron diferencias estadísticamente significativas donde la subregión con mayor porcentaje de incidencia minera (actividad y producción) fue San Juan, seguida en orden de importancia por Medio Atrato, Litoral del Pacífico, Alto Atrato y Bajo Atrato (cuadro 2).

Registros de especies y estado de conservación. Actualmente el Chocó cuenta con registros de 5.976 especies de plantas, habiéndose reportado los mayores porcentajes de ellas en los municipios de Bahía Solano (22,1\%), Quibdó (21,7 \%), San José del Palmar (20,3 \%), Nuquí (16,0 \%), Riosucio (15,9\%) y Acandí (14,8 \%). Los 24 municipios 
Cuadro 1. Vulnerabilidad florística potencial (IVFP) frente a la minería auroplatinífera en los municipios del Chocó, Colombia. IAMAR = índice de actividad minera auroplatinífera relativa, IPMAR = índice de producción auroplatinífera relativa, EDR = especies registradas solo para el Chocó, EA= especies incluidas en alguna categoría de amenaza.

Potential ecological vulnerability (IVFP) related to gold and platinum mining in the municipalities of Chocó, Colombia. IAMAR $=$ index of relative gold and platinum mining activity, IPMAR $=$ index of relative gold and platinum production, $\mathrm{EDR}=$ species recorded only for Chocó, $\mathrm{EA}=$ endangered species.

\begin{tabular}{|c|c|c|c|c|c|c|c|c|c|c|c|c|c|c|c|}
\hline \multirow{3}{*}{ Municipios } & \multirow{3}{*}{ Subregión } & \multicolumn{12}{|c|}{$\begin{array}{r}\text { Factores } \\
\end{array}$} & \multirow{3}{*}{ IVFP } & \multirow{3}{*}{$(\%)$} \\
\hline & & \multicolumn{4}{|c|}{ Presión sobre el ecosistema } & \multicolumn{8}{|c|}{ Sensibilidad del ecosistema } & & \\
\hline & & IAMAR & $(\%)$ & IPMAR & $(\%)$ & Registros & $(\%)$ & Especies & $(\%)$ & EDR & $(\%)$ & EA & $(\%)$ & & \\
\hline Acandí & Bajo Atrato & 0,00 & 0,00 & 0,07 & 0,04 & 4097 & 9,05 & 886 & 14,8 & 1 & 0,81 & 26 & 4,49 & 5,34 & 1,18 \\
\hline Alto Baudó & Litoral del Pacífico & 0,00 & 0,00 & 4,78 & 2,4 & 508 & 1,12 & 228 & 3,8 & 11 & 8,94 & 1 & 0,17 & 11,50 & 2,55 \\
\hline Atrato & Medio Atrato & 8,60 & 2,87 & 7,34 & 3,7 & 579 & 1,28 & 221 & 3,7 & 7 & 5,69 & 2 & 0,35 & 12,60 & 2,78 \\
\hline Bagadó & Alto Atrato & 8,35 & 2,78 & 1,26 & 0,6 & 23 & 0,05 & 19 & 0,3 & 0 & 0,00 & 0 & 0,00 & 3,41 & 0,76 \\
\hline Bahía Solano & Litoral del Pacífico & 3,74 & 1,25 & 0,00012 & 0,00006 & 5345 & 11,81 & 1319 & 22,1 & 13 & 10,6 & 47 & 8,12 & 19,90 & 4,41 \\
\hline Bajo Baudó & Litoral del Pacífico & 14,0 & 4,67 & 0,018 & 0,009 & 799 & 1,77 & 495 & 8,3 & 1 & 0,81 & 43 & 7,43 & 12,90 & 2,86 \\
\hline Bojayá & Medio Atrato & 7,48 & 2,49 & 0,71 & 0,36 & 350 & 0,77 & 287 & 4,8 & 2 & 1,63 & 39 & 6,74 & 11,20 & 2,48 \\
\hline Cantón de San Pablo & San Juan & 37,2 & 12,4 & 11,6 & 5,82 & 60 & 0,13 & 0 & 0,0 & 0 & 0,00 & 0 & 0,00 & 18,20 & 4,03 \\
\hline Carmen de Atrato & Alto Atrato & 0,93 & 0,31 & 1,22 & 0,61 & 546 & 1,21 & 213 & 3,6 & 8 & 6,50 & 13 & 2,25 & 9,67 & 2,14 \\
\hline Carmen del Darién & Bajo Atrato & 0,00 & 0,00 & 0,00 & 0,00 & 2 & 0,004 & 2 & 0,03 & 1 & 0,81 & 0 & 0,00 & 0,81 & 0,18 \\
\hline Cértegui & San Juan & 2,60 & 0,87 & 12,5 & 6,23 & 103 & 0,23 & 314 & 5,3 & 6 & 4,88 & 18 & 3,11 & 15,10 & 3,34 \\
\hline Condoto & San Juan & 47,5 & 15,8 & 36,0 & 18,0 & 167 & 0,37 & 40 & 0,7 & 7 & 5,69 & 18 & 3,11 & 42,60 & 9,43 \\
\hline Istmina & San Juan & 24,7 & 8,24 & 20,0 & 10,0 & 628 & 1,39 & 321 & 5,4 & 13 & 10,6 & 36 & 6,22 & 35,00 & 7,75 \\
\hline Juradó & Litoral del Pacífico & 2,80 & 0,93 & 0,00 & 0,00 & 165 & 0,36 & 47 & 0,8 & 0 & 0,00 & 3 & 0,52 & 1,45 & 0,32 \\
\hline Litoral del San Juan & San Juan & 0,00 & 0,00 & 0,01 & 0,005 & 232 & 0,51 & 125 & 2,1 & 6 & 4,88 & 25 & 4,32 & 9,20 & 2,04 \\
\hline Lloró & Alto Atrato & 17,8 & 5,93 & 2,28 & 1,14 & 1176 & 2,60 & 146 & 2,4 & 1 & 0,81 & 3 & 0,52 & 8,40 & 1,86 \\
\hline Medio Atrato & Medio Atrato & 4,49 & 1,50 & 1,92 & 0,96 & 282 & 0,62 & 61 & 1,0 & 1 & 0,81 & 0 & 0,00 & 3,27 & 0,72 \\
\hline Medio Baudó & Litoral del Pacífico & 0,00 & 0,00 & 14,3 & 7,13 & 667 & 1,47 & 203 & 3,4 & 0 & 0,00 & 0 & 0,00 & 7,13 & 1,58 \\
\hline Medio San Juan & San Juan & 1,38 & 0,46 & 6,1 & 3,05 & 54 & 0,12 & 50 & 0,8 & 2 & 1,63 & 0 & 0,00 & 5,14 & 1,14 \\
\hline Nóvita & San Juan & 22,4 & 7,47 & 27,3 & 13,6 & 536 & 1,18 & 360 & 6,0 & 13 & 10,60 & 19 & 3,28 & 34,90 & 7,74 \\
\hline Nuquí & Litoral del Pacífico & 2,80 & 0,93 & 0,00 & 0,00 & 8091 & 17,88 & 958 & 16,0 & 19 & 15,40 & 47 & 8,12 & 24,50 & 5,42 \\
\hline Quibdó & Medio Atrato & 13,9 & 4,63 & 7,78 & 3,89 & 6760 & 14,94 & 1296 & 21,7 & 15 & 12,20 & 80 & 13,80 & 34,50 & 7,64 \\
\hline Río Iró & San Juan & 3,97 & 1,32 & 3,36 & 1,68 & 2 & 0,004 & 1 & 0,02 & 0 & 0,00 & 1 & 0,17 & 3,18 & 0,70 \\
\hline Río Quito & Medio Atrato & 11,2 & 3,73 & 0,87 & 0,43 & 185 & 0,41 & 37 & 0,6 & 1 & 0,81 & 5 & 0,86 & 5,84 & 1,29 \\
\hline Riosucio & Bajo Atrato & 5,61 & 1,87 & 1,48 & 0,74 & 7017 & 15,51 & 951 & 15,9 & 3 & 2,44 & 98 & 16,90 & 22,00 & 4,86 \\
\hline San José del Palmar & San Juan & 0,00 & 0,00 & 1,14 & 0,57 & 4083 & 9,02 & 1211 & 20,3 & 30 & 24,4 & 27 & 4,66 & 29,60 & 6,56 \\
\hline Sipí & San Juan & 19,8 & 6,61 & 9,05 & 4,53 & 71 & 0,16 & 44 & 0,7 & 1 & 0,81 & 0 & 0,00 & 11,90 & 2,65 \\
\hline Tadó & San Juan & 22,4 & 7,47 & 12,4 & 6,21 & 1128 & 2,49 & 369 & 6,2 & 5 & 4,07 & 13 & 2,25 & 20,00 & 4,43 \\
\hline Unguía & Bajo Atrato & 2,48 & 0,83 & 0,02 & 0,01 & 687 & 1,52 & 69 & 1,2 & 1 & 0,81 & 46 & 7,94 & 9,59 & 2,12 \\
\hline Unión Panamericana & San Juan & 13,9 & 4,63 & 16,60 & 8,30 & 909 & 2,01 & 314 & 5,3 & 11 & 8,94 & 5 & 0,86 & 22,70 & 5,03 \\
\hline Total & & 300 & 100 & 200 & 100 & 45252 & 100 & 5976 & 177 & 123 & 146 & 579 & 106 & 452 & 100 \\
\hline Chi-cuadrado & & & & & & 106831,9 & & 16354,4 & & 243,1 & & 880,3 & & & \\
\hline$P$ & & & & & & $<0,0001$ & & $<0,0001$ & & $<0,0001$ & & $<0,0001$ & & & \\
\hline
\end{tabular}


Cuadro 2. Vulnerabilidad florística potencial (IVFP) frente a la minería auroplatinífera en las cinco subregiones ecogeográficas del Chocó, Colombia. IAMAR = índice de actividad minera auroplatinífera relativa, IPMAR = índice de producción auroplatinífera relativa, EDR = especies registradas solo para el Chocó, EA = especies incluidas en alguna categoría de amenaza.

Potential ecological vulnerability (IVFP) related to gold and platinum mining in the five ecogeographic sub-regions of Chocó, Colombia. IAMAR = index of relative gold and platinum mining activity, IPMAR $=$ index of relative gold and platinum production, $E D R=$ species recorded only for Chocó, EA = endangered species.

\begin{tabular}{|c|c|c|c|c|c|c|c|c|c|c|c|c|c|c|}
\hline \multirow{3}{*}{ Subregión } & \multicolumn{12}{|c|}{ Parámetros } & \multirow{3}{*}{ IVFP } & \multirow{3}{*}{$(\%)$} \\
\hline & \multicolumn{4}{|c|}{ Presión sobre el ecosistema } & \multicolumn{8}{|c|}{ Sensibilidad del ecosistema } & & \\
\hline & IAMAR & $(\%)$ & IPMAR & $(\%)$ & Registros & $(\%)$ & Especies & $(\%)$ & EDR & $(\%)$ & EA & $(\%)$ & & \\
\hline Bajo Atrato & 8,09 & 2,70 & 1,57 & 0,78 & 11803 & 26 & 1512 & 25,30 & 6 & 4,88 & 170 & 29,40 & 37,7 & 8,30 \\
\hline Medio Atrato & 45,60 & 15,20 & 18,60 & 9,31 & 8156 & 18 & 1362 & 22,80 & 26 & 21,10 & 126 & 21,80 & 67,4 & 14,90 \\
\hline Alto Atrato & 27,01 & 9,02 & 4,76 & 2,38 & 1745 & 4 & 362 & 6,06 & 9 & 7,32 & 16 & 2,76 & 21,5 & 4,76 \\
\hline San Juan & 196,00 & 65,30 & 156 & 78,00 & 7973 & 18 & 1980 & 33,10 & 94 & 76,40 & 162 & 28,00 & 248,0 & 54,80 \\
\hline Litoral del Pacífico & 23,40 & 7,79 & 19,10 & 9,53 & 15575 & 34 & 2454 & 41,10 & 44 & 35,80 & 141 & 24,40 & 77,4 & 17,10 \\
\hline Total & 300 & 100 & 200 & 100 & 45252 & 100 & 5976 & 128 & 123 & 146 & 579 & 106 & 452 & 100 \\
\hline Chi-cuadrado & & & & & 11654,3 & & 1596,4 & & 144,0 & & 126,1 & & & \\
\hline$P$ & & & & & $<0,0001$ & & $<0,0001$ & & $<0,0001$ & & $<0,0001$ & & & \\
\hline
\end{tabular}


restantes, con excepción de Cantón de San Pablo donde no se encontraron registros, mostraron valores inferiores al $10 \%$ (entre $0,02 \%$ en Río Iró y 8,3 \% en Bajo Baudó; cuadro 1). En este punto, es importante destacar que el bajo número de especies registradas en algunos entes territoriales podría aumentar con la realización de inventarios de biodiversidad en esos territorios.

De las 5.976 especies de plantas registradas para el Chocó, $579(9,7 \%)$ se encuentran catalogadas dentro de alguna categoría de amenaza, y su representatividad varió significativamente entre municipios y subregiones (cuadros 1 y 2 ). Riosucio (16,9\%), Quibdó (13,8 \%), Bahía Solano (8,1 \%), Nuquí $(8,1 \%)$ y Unguía $(7,9 \%)$ presentaron el mayor número de especies bajo alguna categoría de amenaza. El resto de municipios, con excepción de Medio Baudó, Medio San Juan, Medio Atrato, Bagadó y Carmen del Darién, donde no se encontraron reportes de especies amenazadas, mostraron valores entre $0,2 \%$ en Juradó y 7,4 \% en Bajo Baudó (cuadro 1). A escala subregional, la mayor riqueza de especies fue registrada en el Litoral del San del Pacífico, seguida por el San Juan, Bajo Atrato, Medio Atrato y Alto Atrato. Sin embargo, cuando se trató de especies amenazadas, el Bajo Atrato es la subregión más representativa, seguida por San Juan, Litoral Pacífico, Medio Atrato y Alto Atrato (cuadro 2). La proporción de especies en peligro de extinción en relación a la riqueza total de especie reportadas fue mayor en Bajo Atrato (11,8 \%) que en las otras subregiones (San Juan $=8,74 \%$, Medio Atrato $=8,59 \%$, Litoral Pacífico $=5,54 \%$, Alto Atrato $=4,14 \%$ ).

Al analizar la distribución de las 123 especies endémicas de Colombia restringidas geográficamente al Chocó biogeográfico, se pudieron detectar diferencias entre los municipios y las subregiones (cuadros 1 y 2). Con excepción de Bagadó, Cantón de San Pablo, Juradó, Medio Baudó y Río Iró, donde no se encontraron registros, los municipios de San José del Palmar (24,4 \%), Nuquí (15,4 \%), Quibdó (12,2\%), Bahía Solano (10,6 \%), Istmina (10,6 \%) y Nóvita $(10,6 \%)$ fueron los entes territoriales donde más especies de distribución restringida fueron reportadas (cuadro 1). A escala subregional, San Juan, Litoral del Pacífico y Medio Atrato mostraron mayor representatividad en riqueza de especies restringidas geográficamente al Chocó (cuadro 2).

Vulnerabilidad potencial para la pérdida de especies. El grado de vulnerabilidad florística potencial (pérdida potencial de especies) frente a la minería auroplatinífera realizada con maquinaria pesada en el Chocó, varió significativamente entre municipios y subregiones (cuadros 1 y 2). A nivel municipal, Condoto (9,43\%), Istmina (7,75\%), Nóvita (7,74 \%), Quibdó (7,64 \%) y San José del Palmar $(6,56 \%)$ fueron los municipios con mayor vulnerabilidad florística potencial (cuadro 1). A nivel subregional, San Juan presentó el mayor porcentaje de vulnerabilidad, seguido en orden descendente por Litoral del Pacífico, Medio Atrato, Bajo Atrato y Alto Atrato (cuadro 2).
El análisis de componentes principales mostró, además, que los entes territoriales (municipios y subregiones) difieren en cuanto a la contribución relativa de los distintos componentes de vulnerabilidad. En San José del Palmar, Nuquí, Quibdó, Bahía Solano, Riosucio y Alto Baudó el grado de vulnerabilidad está más relacionado con el número de especies amenazadas y de distribución restringida que con la presencia misma de la actividad minera en esos territorios (figura 4A). Por su parte, en la mayoría de los municipios de la subregión del San Juan (e.g. Condoto, Istmina, Unión Panamericana y Tadó) la vulnerabilidad está
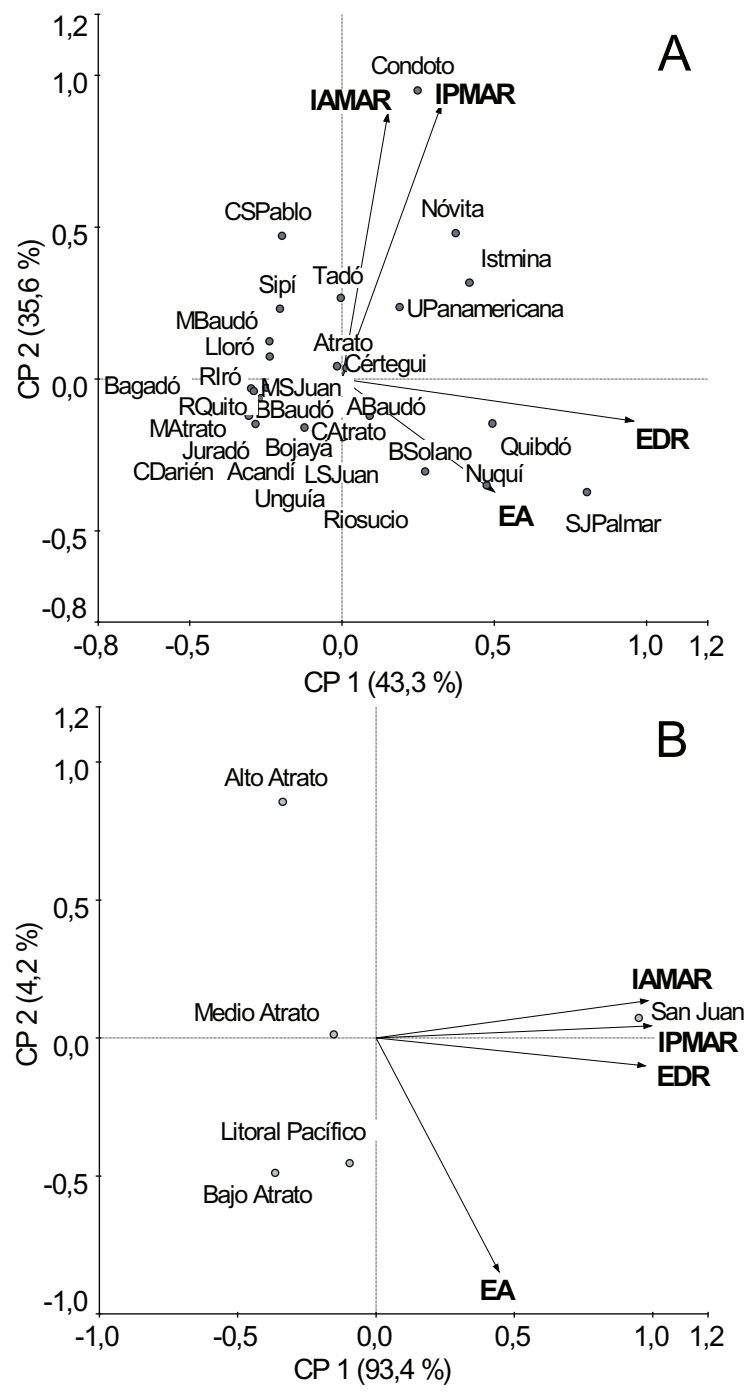

Figura 4. Relación entre las variables de incidencia minera (IAMAR, IPMAR) y la sensibilidad ecosistémica (EA y EDR) en los municipios (A) y las subregiones (B) del Chocó, Colombia. Los valores en porcentaje indican la varianza explicada por cada componente principal.

Relationship among variables of mining incidence (IAMAR, IPMAR) and floristic sensitivity (EA and EDR) in the municipalities (A) and sub-regions (B) of Chocó, Colombia. Percentage values indicate the variance explained by each main component. 
más ligada a la incidencia de la minería y a la cantidad de especies de distribución restringida (figura 4A-B). Municipios como Belén de Bajará, Carmen del Darién, Unguía, Medio San Juan, Río Iró, Juradó, Medio Atrato, Bagadó se ordenaron hacia la parte negativa de ambos componentes principales, mostrando poca relación con los factores que definen la vulnerabilidad florística potencial y, en consecuencia, mostrando un bajo potencial para la pérdida de especies por acción de la minería (cuadro 1, figura 4A). Estos resultados indican que las selvas de entes territoriales pertenecientes a la subregión del San Juan se encuentran más amenazados, desde el punto de vista de la posible pérdida de especies por la incidencia de la minería, que los municipios de otras subregiones del Chocó.

\section{DISCUSIÓN}

Los resultados de este estudio constatan que el Chocó contribuye sustancialmente a la producción auroplatinífera de Colombia, aunque más del $90 \%$ de la actividad minera desarrollada en él es informal e ilegal (Guiza 2013), y hace uso mayoritariamente de maquinaria pesada para incrementar la producción.

Aunque en este estudio no se cuantifican de manera directa los impactos de la minería sobre el bosque tropical, algunos autores indican que la minería con maquinaria pesada genera reducción de la belleza estética del paisaje, pérdida de hábitats y conectividad de la cubierta vegetal, erosión del suelo y contaminación de ríos (Ramírez-Moreno y Ledezma-Rentería 2007). Todos estos impactos, sumados a la promoción de políticas de estímulo a la ocupación minera y al desconocimiento del potencial estratégico de la biodiversidad de los bosques en cuyo seno se desarrolla la minería, están contribuyendo a la pérdida de biodiversidad en Colombia (Andrade-C 2011).

A pesar de los graves impactos de la minería sobre la diversidad de especies, esta actividad se practica en más del $90 \%$ de los municipios del Chocó. No obstante, la actividad minera se concentra en la subregión del San Juan, cuyos municipios registran los valores más altos de vulnerabilidad por presentar un alto número de especies de distribución restringida y en peligro de extinción, además de alta incidencia de la actividad minera. Estos resultados ponen de manifiesto la necesidad de aplicar medidas de protección, conservación y/o restauración de aquellos sistemas forestales en zonas con alta vulnerabilidad, como es el caso de la subregión del San Juan en comparación con los municipios de otras regiones del Chocó.

Las acciones que se podrán emprender para hacer de la minería una actividad socialmente más responsable con la conservación de la naturaleza son variadas, y se podrían aplicar enfoques diversificados: e.g. en zonas donde el grado de vulnerabilidad sea más alto, las medidas deberían procurar por el mantenimiento de la composición, estructura y función de áreas sin actividad minera, así como la restauración ecológica de áreas impactadas por ella. Mien- tras que en zonas donde el grado de vulnerabilidad sea más bajo, las medidas deberían implicar el mantenimiento de los ecosistemas, así como de los procesos ecológicos que los sustentan.

A nivel internacional, la puesta en marcha de acciones conservacionistas tiene una fundamentación de carácter legal, ambiental y social, pero ¿cuenta el Chocó y los entes territoriales estudiados con cualidades que permitan definir medidas de protección de la biodiversidad? Según el Decreto 2372 de 1 de julio de 2010, del Ministerio de Ambiente Vivienda y Desarrollo Territorial de la república de Colombia, las áreas protegidas son el elemento central para la conservación de la diversidad biológica en Colombia (Artículo 4), y sus objetivos están centrados en preservar, restaurar y mantener los ecosistemas naturales y los procesos evolutivos y dinámicos que los sustentan (Artículo 6). En particular, el territorio chocoano forma parte de una de las 25 áreas priorizadas a nivel global (hotspot) para la conservación de la biodiversidad (Myers et al. 2000, Primack et al. 2001), pues además de ser una región rica en biodiversidad y especies endémicas (Gentry 1986, Bernal et al. 2015), su biota se encuentra amenazada por actividades como la minería (Andrade-C 2011). Por lo tanto, el establecimiento de figuras de protección de la biodiversidad es viable en términos legales, ambientales y sociales en el territorio. El gran reto estaría en determinar cómo establecer prioridades de conservación y categoría de protección dentro del territorio.

En Colombia existen varias categorías de áreas protegidas según las particularidades de las zonas a conservar y los fines de gestión (Decreto 2372 de 2010): 1) Parques Nacionales Naturales (Decreto Ley 2811 de 1974) que incluyen, a su vez: a) Parque nacional (área donde los ecosistemas no han sido alterados sustancialmente y sus propiedades tienen valor científico, educativo, estético y recreativo Nacional); b) Reserva natural (área prístinas destinadas a la conservación y estudio de sus riqueza natural); c) Área natural única (área de condiciones especiales que merece ser conservada); d) Santuario de flora (área dedicada a preservar la flora Nacional); e) Santuario de fauna (área dedicada a preservar la fauna Nacional); y f) Vía parque (faja de terreno con carretera con bellezas escénica, valores naturales o culturales para fines de educación y esparcimiento). 2) Reservas forestales protectoras: Área donde los bosques mantienen su función, aunque su estructura y composición haya sido modificada. 3) Parques naturales regionales: Área donde los ecosistemas mantienen la estructura, composición y función, y los procesos ecológicos y evolutivos que los sustentan. 4) Distritos de manejo integrado: Área donde los ecosistemas mantienen su composición y función, aunque su estructura haya sido modificada. 5) Distritos de conservación de suelos. Área donde los ecosistemas mantienen su función, aunque su estructura y composición hayan sido modificadas. 6) Áreas de recreación. Área donde los ecosistemas mantienen su función, aunque su estructura y composición hayan sido 
modificadas. 7) Reservas naturales de la sociedad civil. Área que conserva una muestra de un ecosistema natural y que por la voluntad de su propietario se destina a la protección. Es importante resaltar que las áreas protegidas de la 2 a la 7 , se ponen al alcance de los seres humanos para su conocimiento, conservación, restauración y disfrute.

Partiendo de las anteriores definiciones y del grado de vulnerabilidad de los entes territoriales, una aproximación para priorizar áreas y figuras de conservación en el Chocó puede ser la que se describe a continuación: En territorios con menor vulnerabilidad se podrían establecer parques naturales, reserva natural o santuarios de flora y fauna, pues estas figuras no implican el uso de los predios más allá del disfrute escénico. Mientras que en territorios con mayor vulnerabilidad se puede implementar una combinación de figuras: por un lado, se pueden establecer parques naturales regionales o santuarios de flora y fauna en sectores donde los ecosistemas mantengan sus propiedades naturales, y por otro, distritos regionales de manejo integrado, áreas de recreación, distritos de conservación de suelos o reservas naturales de la sociedad civil en sectores destinados para acciones mineras, ya que estas figuras consideran el uso de los predios declarados más allá del disfrute escénico.

Además de la declaración de las distintas figuras de protección, la aplicación de programas de restauración ecológica constituye una estrategia para mantener la biodiversidad en regiones de alta vulnerabilidad. Actualmente existen pocas experiencias sobre restauración ecológica en la región del Chocó. Sin embargo, la repoblación de minas con especies exóticas es inminente en esta región (Ayala et al. 2008), desconociendo sus efectos sobre la sucesión natural (Murcia y Guariguata 2014). A pesar de ello, algunos investigadores sugieren que la calidad de sitio (MartínezRamos y García-Orth 2007), el tipo de plantas y el clima (Alday et al. 2010); la fertilidad del suelo y el banco semillas (DeFalco et al. 2012), así como también, la edad y la distancia del área afectada respecto a la matriz de vegetación remanente (Martín-Sanz et al. 2015), son factores que explican la regeneración natural de zonas donde la sucesión primaria es el punto de partida. Además, el éxito de un programa de restauración ecológica depende de las particularidades biofísicas de cada región. En este sentido, el Chocó cuenta con una serie de atributos que la hacen especial, pues además de su exuberante diversidad biológica, cuenta con uno de los regímenes de precipitación más altos del mundo, lo cual no garantiza la eficacia de protocolos exitosos en otras latitudes.

\section{CONCLUSIONES}

El auge de la minería auroplatinifera es un factor de amenaza para el mantenimiento de la vida silvestre en regiones tropicales con alta biodiversidad, que enfatiza la necesidad de establecer áreas protegidas y programas de restauración ecológica en territorios con alta vulne- rabilidad florística, como los del San Juan en el Chocó biogeográfico y otras regiones tropicales mineras y biodiversas, donde el índice de vulnerabilidad florística potencial puede ser adecuadamente replicado. En este punto, es importante destacar que, aunque es un índice eficiente para determinar la vulnerabilidad de los bosques frente a la minería en distintos territorios, se debe tener en cuenta que esta herramienta puede mejorar sus estimaciones a nivel de ecosistema, si se realizan inventarios de biodiversidad completos, cantidad de área afectada y estudios edáficos que permitan adicionar a la ecuación otras variables ecológicas afectadas por la minería como la riqueza del suelo y la diversidad de especies, no solo de la flora nativa, sino también de la fauna que habita los bosques en situación de vulnerabilidad.

\section{AGRADECIMIENTOS}

A los pobladores de Condoto, Istmina, Río Quito, Tadó y Unión Panamericana (Chocó) por su hospitalidad. A S. Eccehomo por su acompañamiento; Z Quinto, K Valencia, H Quinto, DA Lozano y tres revisores anónimos por sus valiosos comentarios. A Steve Cagan por suministrar las figuras $1 \mathrm{~B}$ y $1 \mathrm{~F}$ (foto (C) Steve Cagan-HYPERLINK "http://www.stevecagan.com/" \t " blank" www.stevecagan.com). Este trabajo fue financiado por la Universidad Tecnológica del Chocó (UTCH) a través del Proyecto Biochocó (Código-BPIN 2013000100191), y por la Fundación Carolina (FC), la UTCH y la Universidad de Valladolid (UVa) a través de una beca asignada a $\mathrm{H}$ Valois.

\section{REFERENCIAS}

Agencia Nacional Minera. 2015. Producción oficial de minerales en Colombia. Consultado 10 jun. 2015. Disponible en http://www.anm.gov.co.

Alday JG, RH Marrs, C Martínez-Ruiz. 2010. The importance of topography and climate on short-term vegetation of coal wastes in Spain. Ecological Engineering 36(4): 579-585. DOI: 10.1016/j.ecoleng.2009.12.005.

Andrade-C G. 2011. Estado del conocimiento de la biodiversidad en Colombia y sus amenazas. Consideraciones para fortalecer la interacción ciencia-política. Revista de la Academia Colombiana de Ciencias Exactas Físicas y Naturales 35(137): 491-507.

Ayala JH, J Mosquera, WI Murillo. 2008. Evaluación de la adaptabilidad de la acacia (Acacia mangium Wild), y bija (Bixa orellana) en áreas degradadas por la actividad minera aluvial en el Chocó biogeográfico, Condoto, Chocó, Colombia. Bioetnia 5(2): 115-123.

Bernal R, SR Gradstein, M Celis. 2015. Catálogo de plantas y líquenes de Colombia. Bogotá, Colombia. Instituto de Ciencias Naturales - Universidad Nacional de Colombia. Consultado 10 jun. 2015. Disponible en http://catalogoplantasdecolombia.unal.edu.co

CODECHOCO (Corporación Autónoma Regional para el Desarrollo Sostenible del Chocó, CO). 2012. Situación actual de la minería en el departamento de Chocó. Quibdó, Colom- 
bia. Subdirección de Calidad y Control Ambiental. 33 p. (Informe de gestión 2012).

DeFalco LA, TC Esque, MB Nicklas, JM Kane. 2012. Supplementing seed banks to rehabilitate disturbed Majove desert shrublands: Where do all the seeds go? Restoration Ecology 20(1): 85-94.

Gentry AH. 1986. Species richness and floristic composition or Chocó region plant communities. Caldasia 15(71-75): 7 $1-91$.

Jones CC, R del Moral. 2005. Patterns of primary succession on the foreland of Coleman Glacier, Washington, USA. Plant Ecology 180(1): 105-116.

Leal C. 2009. La compañía minera Chocó Pacífico y el auge del platino en Colombia, 1897-1930. Historia crítica 1(edición especial):150-164.

Martín-Sanz RC, B Fernández-Santos, C Martínez-Ruiz. 2015. Early dynamics of natural revegetation on roadcuts of the Salamanca province (CW Spain). Ecological Engineering 75: 223-231. DOI: 10.1016/j.ecoleng.2014.11.057.

Martínez-Ramos M, X García-Orth. 2007. Sucesión ecológica y restauración de las selvas húmedas. Boletín de la Sociedad Botánica de México 80(suplemento): 69-87.

Murcia C, MR Guariguata. 2014. La restauración ecológica en Colombia: Tendencias, necesidades y oportunidades. Bogor, Indonesia. CIFOR. 65 p. (Documentos Ocasionales 107).

Myers N, RA Mittermeier, CG Mittermeier, GAB da Fonseca, J Kent. 2000. Biodiversity hotspots for conservation priorities. Nature 403(6772): 853-858. DOI: 10.1038/35002501.

NYBG (The New York Botanical Garden, US). 2015. The C. V. Starr Virtual Herbarium. The New York Botanical Garden. Consultado 20 jun. 2015. Disponible en http://sweetgum. nybg.org/science/vh/.

Poveda-M IC, CA Rojas-P, A Rudass-LI, JO Rangel-Ch. 2004. El Chocó biogeográfico: ambiente físico. In Rangel-Ch JO ed. Colombia diversidad biótica IV - El Chocó biogeográfico/ Costa Pacífica. Bogotá, Colombia. Universidad Nacional de Colombia-Conservación Internacional. p. 1-22.

Primack R, R Rozzi, P Feinsinger. 2001. XV. Establecimiento de áreas protegidas. In Primack R, R Rozzi, P Feinsinger, R Dirzo, F Massardo eds. Fundamentos de conservación biológica: perspectivas latinoamericanas. México DF, México.
Fondo de Cultura Económica. p. 449-476.

R Core Team. 2012. R: A language and environment for statistical computing. R Foundation for Statistical Computing, Vienna, Austria. Consultado 10 jun. 2015. Disponible en http://www.R-project.org/

Ramírez-Moreno G, E Ledezma-Rentería. 2007. Efecto de las actividades socioeconómicas (minería y explotación maderera) sobre los bosques del departamento del Chocó. Revista Institucional Universidad Tecnológica del Chocó 26: 58-65.

Rangel-Ch. 2004a. Colombia diversidad biótica IV - El Chocó biogeográfico/Costa Pacífica. Bogotá, Colombia. Universidad Nacional de Colombia-Conservación Internacional. $997 \mathrm{p}$.

Rangel-Ch. 2004b. Amenazas a la biota y a los ecosistemas del Chocó biogeográfico. In Rangel-Ch JO ed. Colombia diversidad biótica IV - El Chocó biogeográfico/Costa Pacífica. Bogotá, Colombia. Universidad Nacional de ColombiaConservación Internacional. p. 841-866.

Rangel-Ch JO, O Rivera-Díaz. 2004. Diversidad y riqueza de espermatofitos en el Chocó biogeográfico. In Rangel-Ch JO ed. Colombia diversidad biótica IV - El Chocó biogeográfico/Costa Pacífica. Bogotá, Colombia. Universidad Nacional de Colombia-Conservación Internacional. p. 83-14.

Rangel-Ch JO, O Rivera-Díaz, D Giraldo-Cañas, C Parra-O, JC Murillo-A, I Gil, JL Fernández, J Sarmiento, G Galeano, R. Bernal, S Suárez, JR Botina, ME Morales, C Berg. 2004. Catálogo de espermatofitos en el Chocó biogeográfico. In Rangel-Ch JO ed. Colombia diversidad biótica IV - El Chocó biogeográfico/Costa Pacífica. Bogotá, Colombia. Universidad Nacional de Colombia-Conservación Internacional. p. 105-439.

SiB Colombia. 2015. Portal de datos SiB Colombia. Consultado 10 jun. 2015. Disponible en http://www.sibcolombia.net/ web/sib/home

Ter Braak CJF, P Šmilauer. 2002. CANOCO Reference manual and CANODRAW for Windows User's Guide version 4.5. Nueva York, USA. Microcomputer Power. 500 p.

Tropicos. 2015. Tropicos.org. Missouri Botanical Garden. Consultado 20 jun. 2015. Disponible en http://www.tropicos. org. 
